\title{
EDITORIALE
}

\section{MARKETING STRATEGICO E TIPICITÀ LOCALI}

\author{
Aldo Burresi *
}

Recenti fatti economici dimostrano come uno degli effetti più dirompenti della globalizzazione è rappresentato dallo spostamento di intere imprese (gli stabilimenti ed il suo vertice) dal paese di origine a paesi di nuova industrializzazione contraddistinti da un minor costo della manodopera e da condizioni di lavoro più favorevoli. Di fronte a tali dinamiche le istituzioni politiche e sindacali del paese di origine dell'impresa mostrano di avere uno scarso potere di condizionamento e di freno ed in ogni caso sono costrette a contrattare in una posizione di debolezza in quanto la sopravvivenza dell'impresa nello scacchiere competitivo globale sembra non consentire altre soluzioni. In questi contesti di mercato caratterizzati da una globalizzazione sempre più spinta (Valdani, 2009) e da un appiattimento ed un'omologazione dell'offerta, con il rischio di una perdita della tipicità propria dei prodotti e dei paesi d'origine il marketing strategico, nella misura in cui vi sia una condivisione da parte del vertice, potrebbe costituire, a nostro avviso, uno strumento fondamentale per recuperare a livello di impresa e di paese i vantaggi competitivi basati sulle tradizioni, le competenze e le tipicità quali espressioni di un territorio (Caroli, 2007). Ciò comporterà che a livello di impresa si valorizzino e si sviluppino ulteriormente le competenze proprie del marketing strategico e che a livello territoriale, si assumano iniziative collettive spinte anche da organismi istituzionali capaci di coinvolgere le imprese e di generare un raccordo con il mercato e con i paesi di destinazione. In questo contesto uno strumento fondamentale di valorizzazione sia delle imprese che del paese di origine è la marca quale potenziale sintesi dell'identità dell'impresa e del paese.

* Professore Ordinario di Marketing presso 1'Università degli Studi di Firenze; e-mail: aldo.burresi@unifi.it.

Mercati e Competitività n. 2, 2011 


\section{A. Burresi}

Nei mercati si assiste alla presenza di una sempre maggiore distanza sia fisica che psicologica tra l'offerta, realizzata in contesti geografici molto lontani dal mercato di sbocco, e la domanda che localizzata in paesi diversi e con differenti motivazioni di acquisto, ha spesso un'idea di prodotto generica e pertanto tende a ricercare nell'offerta elementi di omogeneità. In altri termini l'offerta, pur di fronte ad una domanda variegata, è spinta ad adattarsi alla domanda con un prodotto tendente all'omogeneizzazione. Si rileva dunque da parte dell'impresa la necessità di intervenire sulla riconfigurazione degli equilibri operativi, sulla ridefinizione, all'interno della catena del valore, delle relazioni tra progettazione, produzione, distribuzione fisica e commercializzazione (Chao, 2001). La progettazione, per il suo elevato valore aggiunto, è sovente centralizzata nel paese di origine dell'impresa; la produzione può essere in parte realizzata nel paese di origine ed in parte esternalizzata in aziende di proprietà ed in aziende conto terzi operanti in mercati esteri; la distribuzione e la commercializzazione si collocano sempre più vicino ai mercati di sbocco. Nell'ambito di un approccio standardizzato al mercato, le imprese che operano in contesti globali possono assumere due comportamenti strategici alternativi: alcune tendono a ricreare un ambiente competitivo di impresa andando a cogliere nei contesti globali fondamentali fattori di convergenza, altre impongono la loro offerta sui mercati avvalendosi di rapporti sviluppati con gli intermediari, avviando successivamente un processo di progressivo adattamento al mercato.

Alla luce dei più recenti cambiamenti che coinvolgono sia le imprese, destinate ad operare in contesti sempre più competitivi, che i mercati caratterizzati anche da segmenti di consumatori sensibili alla dimensione territoriale quale attributo dei prodotti, diventa indispensabile riscoprire i fattori distintivi dei territori valorizzando in modo aggregato le loro tipicità, $i$ loro saperi, le competenze stratificate ed accumulate nel tempo, trasformandole in effettive fonti di vantaggio competitivo territoriale. Diventa così rilevante chiederci quale sia il ruolo ed i caratteri che la dimensione strategica del marketing viene ad assumere (Collesei, 2010). Una presenza attiva del marketing strategico implica inevitabilmente la convinzione da parte del vertice di sviluppare un orientamento al mercato basato non solo sulla centralità della domanda finale (Guercini, 2003), ma anche sulla valorizzazione combinata di alcune tipicità territoriali. Questo richiede una rilettura del territorio di origine come produttore di specificità da valorizzare nei mercati internazionali e la capacità di combinare l'effetto paese di origine con l'identità di marca. Si crea così un rapporto biunivoco tra l'offerta dell'impresa ed il territorio: quest'ultimo diventa una componente del prodotto e, allo stesso tempo, un elemento di attrazione esterna e quindi fonte di esperenzialità. Le grandi imprese possono alimentare in maniera autonoma questo rapporto biunivoco e trasformarlo in un vantaggio com- 
petitivo reale per operare su nuovi mercati e su nuovi segmenti. Le azienda di piccole e medie dimensione, invece, che spesso non sono dotate di competenze analitiche sufficienti ad alimentare il rapporto biunivoco individuato, anche se detengono delle specificità, possono essere penalizzate nello sfruttare il territorio come risorsa strategica distintiva; avvertono pertanto l'esigenza di sviluppare accordi ed alleanze con altre imprese del territorio avvalendosi anche del supporto degli organismi istituzionali. In ogni caso anche per le grandi imprese, le istituzioni possono assumere un ruolo di attivatori e di coordinatori di attività volte a far diventare il territorio un oggetto di scambio tra imprese e mercati.

A livello organizzativo questo presuppone la presenza autorevole del marketing nella sua valenza strategica; tale valenza non si basa soltanto su contenuti analitici per quanto sofisticati essi possano essere, ma richiede anche lo sviluppo di relazioni durevoli con gli ambienti istituzionali. In altri termini chi si occupa di marketing strategico si trova a dover interpretare meccanismi burocratico-amministrativi e curare relazioni politico-istituzionali, fondamentali per poter conoscere ed accedere a nuovi mercati. Tali meccanismi e tali relazioni sono propri sia del paese di origine che del paese di destinazione; nei confronti di entrambi si rivolge l'attenzione di chi sovrintendente e svolge attività di marketing strategico. Si realizza quindi una compenetrazione tra la dimensione analitica del mercato e la dimensione politico-istituzionale dei paesi coinvolti.

La marca può costituire un strumento essenziale per valorizzare l'identità e quindi anche le tipicità sia di un territorio che di un paese (Han, 1989). Essa infatti incorpora valori che richiamano le tradizioni, i saperi propri dell'identità territoriale, innescando in questo modo un meccanismo di valorizzazione incrociata nel senso che la marca può diventare l'ambasciatore dell'identità di un paese che, in modo reciproco, è in grado di valorizzare la brand identity. In altri termini, da un lato, l'insieme di marche appartenenti ad un determinato paese possono, attraverso un processo di astrazione, contribuire a definire l'immagine del paese stesso; ciò si verifica anche nel caso in cui il paese di destinazione abbia già un'identità del paese di origine. D'altro lato, una forte identità di quest'ultimo può influire positivamente sulla costruzione della brand identity. Tuttavia una marca legata al paese di origine che non risponda pienamente alle attese del mercato può influire negativamente sull'immagine dello stesso.

La combinazione incrociata tra marca e territorio richiede la presenza di segmenti di mercato, anche ristretti, che conoscono il paese di origine del prodotto e le sue specificità tecnico-produttive e sono sensibili a cogliere ed apprezzare le tipicità di un prodotto-paese; questa include anche un approccio al consumo evoluto ed una disponibilità da parte della domanda a pagare un premium price quale espressione della differenziazione dell'offerta. L'effettiva percezione da parte del mercato di tale combinazione può 


\section{A. Burresi}

determinare nel lungo termine un positivo effetto di contaminazione su tutte le marche del paese appartenenti allo stesso settore. Tale percezione caratterizza, con riferimento alla realtà italiana, soprattutto i prodotti del lusso e della moda (Varaldo, 2009) ed i prodotti enogastronomici. In questo contesto diventa rilevante chiedersi quanto in un ambiente competitivo globale, in cui ci si propone di valorizzare delle identità locali e delle marche rappresentative di tali identità, sia importante preservare accanto al country of design, il country of manufacturing che, in misura più marcata, rappresenta le capacità tecnico-produttive del paese e del suo territorio. Mentre per i prodotti enogastronomici, il country of manufacturing può essere un effettivo elemento distintivo dell'identità di un prodotto e di una marca fino a diventare un elemento di attrazione esperienziale da vivere sul territorio di origine, per i prodotti del lusso e della moda, è il country of design che può assumere un ruolo discriminante nella scelta di acquisto. In ogni caso la concomitanza dei due è un presupposto fondamentale per tutelare le competenze radicate in una nazione ed in determinati territori. Spetta al paese ed alle imprese sviluppare una cultura di consumo orientata alla ricerca di prodotti caratterizzati da una tipicità progettata e realizzata nel paese di origine.

Il marketing strategico, se supportato dal vertice, può diventare una filosofia aziendale in grado di orientare l'impresa verso nuove esperienze nei rapporti tra marca, territorio e cliente. Questo presuppone un marketing manager sensibile nel creare una cultura di consumo sulle tipicità del paese di origine e nello sviluppare con gli organismi istituzionali una condivisione di obiettivi e di risoluzioni tecnico-organizzative volte ad accreditare certe tipicità nei mercati di destinazione. Concretamente il marketing manager, in specifici contesti, pur perseguendo obiettivi di volume e di economicità, deve riuscire ad inserire nell'offerta quegli elementi di tipicità che contraddistinguono un paese. Ne consegue che il marketing strategico, nel contribuire al raggiungimento degli obiettivi economico-finanziari, accresce il ruolo sociale dell'impresa in quanto favorisce la qualificazione ed il mantenimento di determinati tradizioni, saperi e competenze di un territorio nonché preserva equilibri occupazionali interni attraverso la permanenza di filiere integrate. Per di più la valorizzazione dell'identità paese può diventare nel medio-lungo termine per l'impresa una fonte aggiuntiva di redditività. 